\title{
Clinical Aspects of Neonatal Hypoglycemia: A Mini Review
}

\author{
Taygen Edwards and Jane E. Harding* \\ Liggins Institute, University of Auckland, Auckland, New Zealand
}

Introduction: Neonatal hypoglycemia is common and a preventable cause of brain damage. The goal of management is to prevent or minimize brain injury. The purpose of this mini review is to summarize recent advances and current thinking around clinical aspects of transient neonatal hypoglycemia.

Results: The groups of babies at highest risk of hypoglycemia are well defined. However, the optimal frequency and duration of screening for hypoglycemia, as well as the threshold at which treatment would prevent brain injury, remains uncertain. Continuous interstitial glucose monitoring in a research setting provides useful information about

OPEN ACCESS

Edited by:

Paula Caroline Midgley,

University of Edinburgh,

United Kingdom

Reviewed by:

Anastasia Ibba,

Ospedale Microcitemico, Italy

Pratik Shah,

Barts Health NHS Trust,

United Kingdom

*Correspondence:

Jane E. Harding

j.harding@auckland.ac.nz

Specialty section:

This article was submitted to

Pediatric Endocrinology,

a section of the journal

Frontiers in Pediatrics

Received: 14 May 2020 Accepted: 10 December 2020

Published: 08 January 2021

Citation:

Edwards T and Harding JE (2021)

Clinical Aspects of Neonatal

Hypoglycemia: A Mini Review.

Front. Pediatr. 8:562251.

doi: 10.3389/fped.2020.562251 glycemic control, including the duration, frequency, and severity of hypoglycemia. However, it remains unknown whether continuous monitoring is associated with clinical benefits or harms. Oral dextrose gel is increasingly being recommended as a first-line treatment for neonatal hypoglycemia. There is some evidence that even transient and clinically undetected episodes of neonatal hypoglycemia are associated with adverse sequelae, suggesting that prophylaxis should also be considered. Mild transient hypoglycemia is not associated with neurodevelopmental impairment at preschool ages, but is associated with low visual motor and executive function, and with neurodevelopmental impairment and poor literacy and mathematics achievement in later childhood.

Conclusion: Our current management of neonatal hypoglycemia lacks a reliable evidence base. Randomized trials are required to assess the effects of different prophylactic and treatment strategies, but need to be adequately powered to assess outcomes at least to school age.

Keywords: newborn, insulin, screening, diagnosis, continuous glucose monitoring, oral dextrose gel, child development

\section{INTRODUCTION}

Neonatal hypoglycemia is a preventable cause of brain injury. It is common, affecting $5-15 \%$ of all babies (1) and approximately half of at-risk babies (2) and is associated with a range of adverse sequelae $(3,4)$. However, the optimal frequency and duration of screening, as well as the threshold at which treatment would prevent brain injury, remains uncertain. The purpose of this review is to summarize the recent advances in clinical aspects of transient neonatal hypoglycemia. 


\section{PATHOPHYSIOLOGY OF NEONATAL HYPOGLYCEMIA}

Glucose is the primary metabolic fuel for the fetus. The fetus receives glucose from its mother through carrier-mediated diffusion down a concentration gradient across the placenta $(5,6)$. Fetal glucose concentrations are $\sim 80 \%$ of maternal concentrations and fluctuate with changes in maternal glucose concentrations (7). The function of insulin in the fetus is as a growth hormone rather than to regulate glucose concentrations, and secretion of insulin occurs at a lower glucose concentration in the fetus than in postnatal life (8).

Maternal and therefore fetal glucose concentrations increase during labor and delivery in response to secretion of maternal stress hormones such as catecholamines and glucocorticoids (9). Once the umbilical cord is clamped, glucose supply is interrupted and neonatal glucose concentrations decrease, reaching a low point $\sim 1-2 \mathrm{~h}$ after birth. In turn, insulin secretion decreases while secretion of counter-regulatory hormones such as glucagon and catecholamines increases, stimulating gluconeogenesis and glycogenolysis, and resulting in a gradual increase in glucose concentrations (9). However, these do not reach adult concentrations until after $72 \mathrm{~h}$ of age $(10,11)$. Delay or interruption of this postnatal metabolic adaptation results in neonatal hypoglycemia.

Glucose is an essential metabolic fuel for the brain, and in the newborn the proportionately large brain accounts for almost all of total tissue glucose requirements (12). Thus, low glucose concentrations are likely to result in inadequate brain energy supply. Although the newborn brain can use alternative metabolic substrates, the supply of these is limited. Lactate provides a potential alternative fuel in the first $48 \mathrm{~h}$, and ketones may be available on days $3-4$, but each can provide only a small proportion of total brain energy requirements (13).

\section{DEFINING NEONATAL HYPOGLYCEMIA}

The definition of neonatal hypoglycemia remains controversial, and has changed over time (14). However, since the major reason for defining hypoglycemia is to identify a threshold at which treatment would prevent brain injury, an ideal definition would relate to the glucose concentration at which brain function is compromised. This makes a single definition problematic, as the threshold is likely to vary in different babies, depending amongst other things on gestational age, postnatal age, concurrent metabolic demands, co-morbidities and availability of alternative metabolic fuels.

The most widely used definition for neonatal hypoglycemia is a glucose concentration of $<47 \mathrm{mg} / \mathrm{dl}(2.6 \mathrm{mmol} / \mathrm{l})(15-17)$. This arises primarily from two studies published in 1988, which related glucose concentrations to neurological function. One was a retrospective study of 661 preterm babies (birthweight $<1,850 \mathrm{~g}$ ), which reported that a glucose concentration of
TABLE 1 | Risk factors for neonatal hypoglycemia.

Transient neonatal hypoglycemia

Preterm birth

Small or large for dates

Infant of diabetic mother

Perinatal stress (birth asphyxia, hypothermia, respiratory distress, sepsis)

Birth asphyxia

Poor feeding

Maternal use of beta blockers

Antenatal corticosteroids

Persistent neonatal hypoglycemia*

Congenital hyperinsulinism

Hypopituitarism (ACTH deficiency, growth hormone deficiency)

Cortisol deficiency

Glycogen storage disease

Disorders of gluconeogenesis (FBP deficiency, PEPCK deficiency, PC deficiency) Fatty acid oxidation defects

*Occurring after or persisting for $\geq 3$ days (27).

ACTH, Adrenocorticotropic hormone; FBP, Fructose-1,6-bisphophatase; PEPCK, Phosphoenolpyruvate carboxykinase; PC, Pyruvate carboxylase.

$<47 \mathrm{mg} / \mathrm{dl}(2.6 \mathrm{mmol} / \mathrm{l})$ on three or more days was associated with an increased risk of developmental delay at 18 months' corrected age (18). Follow-up of a subgroup showed that reduced motor and arithmetic functioning persisted at 8 years (19).

The second study recorded brainstem or somatosensory evoked potentials in 17 infants, of whom only five were newborns (20). None showed flattening of evoked potentials with a glucose concentration of $>47 \mathrm{mg} / \mathrm{dl}(2.6 \mathrm{mmol} / \mathrm{l})$, although some with a glucose concentration below this still had normal evoked potentials. Both studies concluded that a glucose concentration of $>47 \mathrm{mg} / \mathrm{dl}$ ( $2.6 \mathrm{mmol} / \mathrm{l})$ was likely to be safe.

In situations where evidence-based decisions are not possible, operational thresholds offer a pragmatic guide to clinicians for when intervention may be warranted (1). Screening protocols have recommended different operational thresholds ranging from 18 to $60 \mathrm{mg} / \mathrm{dl}(1.0-3.3 \mathrm{mmol} / \mathrm{l})$ (21-24). However, most recommend aiming for a minimum glucose concentration close to $47 \mathrm{mg} / \mathrm{dl}(2.6 \mathrm{mmol} / \mathrm{l})$ in late preterm and term babies more than a few hours old or requiring treatment.

\section{INCIDENCE AND RISK FACTORS}

The incidence of neonatal hypoglycemia varies between studies depending on the diagnostic threshold, the glucose screening protocol and measurement method used, and the population studied (25). However, the incidence of transient neonatal hypoglycemia is estimated to be $5-15 \%$ of newborns $(1,26)$, and in at-risk babies, it approximates $50 \%$ (2) (Table 1). Babies with multiple risk factors do not have a higher incidence but may experience more severe hypoglycemia. 


\section{MANAGEMENT OF NEONATAL HYPOGLYCEMIA}

\section{Screening for Neonatal Hypoglycemia}

The clinical signs of neonatal hypoglycemia include, but are not limited to, cyanosis, apnea, altered level of consciousness, seizures, lethargy, and poor feeding (24). However, since many of these signs are non-specific, and the majority of babies with low glucose concentrations show no clinical signs, it is recommended that all babies with risk factors undergo regular glucose monitoring.

The optimal frequency and duration of screening remain uncertain. Most protocols recommend screening within 1-4h after birth and then every 3 or $4 \mathrm{~h}$ until euglycemia is maintained over two or three consecutive glucose measurements $(15,21$, $22,24)$. However, all of these guidelines are informed by expert opinion and lack a reliable evidence base (28).

Some specify different monitoring periods dependent on the clinical profile of the baby. For example, the American Academy of Pediatrics recommends that monitoring continues until $12 \mathrm{~h}$ after birth for infants of diabetic mothers or large for gestational age, but for $24 \mathrm{~h}$ for babies who are born late preterm or small for gestational age (21). However, there is no evidence to suggest that cerebral glucose requirements vary between at-risk groups (15).

One study that screened at-risk babies using an accurate glucose oxidase method $1-2 \mathrm{~h}$ after birth then every $3-4 \mathrm{~h}$ before feeds for the first $24 \mathrm{~h}$ and every $3-8 \mathrm{~h}$ from 24 to $48 \mathrm{~h}$ reported no difference between risk groups in the incidence or severity of neonatal hypoglycemia, suggesting that a single screening protocol would be reasonable for all babies at risk (2).

\section{Blood Glucose Monitoring Intermittent Glucose Monitoring}

A common method for measuring glucose concentrations in neonates is by heel-prick blood sampling analyzed using pointof-care non-enzymatic glucometers. These provide quick results at a low cost, are readily available in neonatal units, user-friendly and require small volumes of blood (29).

However, these devices are designed for monitoring high glucose concentrations in diabetics, and are affected by several factors that vary widely in newborns including bilirubin concentrations and hematocrit. They are inaccurate at low glucose concentrations, with estimated false positive and false negative rates of $10-30 \%$, and are not recommended as the sole method for diagnosis of neonatal hypoglycemia $(21,30)$. If pointof-care non-enzymatic glucometers are used for screening, it is critical to confirm the results with a laboratory method (21), but best practice is to use more accurate methods from the start.

Laboratory methods use enzymatic reactions including glucose oxidase, hexokinase or dehydrogenase (29) which are more accurate and sensitive for detecting neonatal hypoglycemia $(31,32)$. However, laboratory methods are costly, take time which can delay prompt intervention, and accuracy is also reliant on the quality of the plasma sample (29). More recent guidelines recommend blood gas analyzers which are quick and accurate if they are immediately available $(15,24)$.
A more feasible alternative in many settings is the newer enzymatic point-of-care analyzers, which have the same accuracy as laboratory methods but the convenience and speed of a cotside measurement. Although they are more expensive per test than the widely used (but inaccurate) test strip glucometers, a recent cost analysis concluded that enzymatic glucometers incurred lower direct costs overall because they avoided the additional costs of retesting in the laboratory (33).

\section{Continuous Interstitial Glucose Monitoring}

Continuous interstitial glucose monitors comprise a sensor placed under the skin, and a recording device, often remote from the sensor, which converts the electrical current generated in the sensor to a glucose concentration using an inbuilt algorithm. Most devices provide a reading every $5 \mathrm{~min}$, giving detailed information about glycemic control including the duration, frequency, and severity of hypoglycemia (34).

Continuous glucose monitors have several limitations. They require calibration against blood glucose concentrations at least every $12 \mathrm{~h}$, so they do not abolish the need for blood tests, and more frequent calibration is recommended for greater accuracy and precision (35). Continuous glucose monitors are also prone to measurement error, and the reading can drift from the calibrated value without detection (35). Because, like point-ofcare glucometers, they are designed for use in diabetes, they are less accurate at low glucose concentrations. The lag period between changes in blood glucose concentrations and changes in the continuous monitor reading is unknown but could be up to $30 \mathrm{~min}$ or more, due both to the time required for glucose to diffuse from blood to interstitial fluid, and to delays built into the algorithms, so that the rapid changes in glucose concentrations that are common in newborn babies are poorly reported by continuous monitors $(36,37)$. Infection at the site of sensor insertion is a theoretical concern, but in practice has rarely been reported, and most studies have reported that sensors can be left in place for a week without complications, even in very low birthweight babies (38).

Most importantly, there is a lack of evidence on whether continuous glucose monitoring is associated with clinical benefits or harms. Continuous glucose monitoring detects many more episodes of low glucose concentrations than does intermittent blood glucose measurement. For example, in 102 babies at risk of hypoglycemia, continuous glucose monitoring identified $11 \%$ more babies and $50 \%$ more episodes of low glucose than intermittent glucose monitoring (39). Others have reported similar differences $(38,40)$. Thus, there is a risk that continuous glucose monitoring may lead to a large increase in diagnosis and treatment, but without evidence that these additional detected episodes are related to brain injury, or that additional treatment will have any long-term benefit.

Despite these limitations, continuous glucose monitoring has enormous potential to improve the management of neonatal hypoglycemia. A randomized trial in 48 very low birthweight babies showed that use of continuous glucose monitoring reduced the number of blood samples taken, detected more episodes of neonatal hypoglycemia and reduced the duration of an episode by half when compared with intermittent 
glucose monitoring (40). Another randomized trial in 50 very preterm babes reported that continuous glucose monitoring in conjunction with an algorithm for glucose infusion titration reduced the duration and severity of hypoglycemic episodes, thereby promoting glycemic stability (41). However, it is not yet known if this improved stability will lead to improved later outcomes.

\section{Treating Neonatal Hypoglycemia}

The goal of treating neonatal hypoglycemia is to prevent or minimize brain injury by maintaining a glucose concentration above an acceptable threshold (25). The usual initial approach is to feed the baby, using either formula or breast milk. When glucose concentrations are $<18-25 \mathrm{mg} / \mathrm{dl}(1.0-1.4 \mathrm{mmol} / \mathrm{l})$ intravenous dextrose (bolus $200 \mathrm{mg} / \mathrm{kg}$ followed by an infusion of around $4-8 \mathrm{mg} / \mathrm{kg}$ per minute) is usually required (21, 24). However, administering intravenous dextrose involves admission to the neonatal intensive care unit (NICU), which is costly, invasive, and separates the mother from her baby, which in turn can increase maternal anxiety and interfere with the establishment of breastfeeding. Severe or prolonged hypoglycemia, indicated by persistently high or ongoing $(\geq 3$ days) intravenous glucose requirements, suggest underlying endocrine or metabolic pathology and further investigation is required (Table 1). Elevated insulin concentrations indicate hyperinsulinism, which suppresses the production of alternative metabolic fuels, and hence maintaining blood glucose $\geq 3.5$ $\mathrm{mmol} / \mathrm{l}$ is recommended (24). Additional treatments, such as diazoxide (42), glucagon $(24,43)$ or glucocorticoids (44) may be required.

Oral dextrose gel $200 \mathrm{mg} / \mathrm{kg}(0.5 \mathrm{ml} / \mathrm{kg}$ of $40 \%$ dextrose), in combination with feeding, is increasingly recommended as a first-line treatment for asymptomatic neonatal hypoglycemia $(45,46)$. A randomized trial of 237 late preterm and term babies at risk of neonatal hypoglycemia $[<47 \mathrm{mg} / \mathrm{dl}(2.6 \mathrm{mmol} / \mathrm{l})]$ demonstrated that compared with feeding alone, $40 \%$ oral dextrose gel $200 \mathrm{mg} / \mathrm{kg}$ plus feeding resulted in fewer treatment failures (hypoglycemia after two treatment attempts), reduced admission to NICU for hypoglycemia and reduced formula feeding at 2 weeks of age (47). A 2-year follow-up established safety by demonstrating similar rates of processing difficulty and neurosensory impairment between the oral dextrose and placebo groups (48). A subsequent cost-utility analysis concluded that dextrose gel resulted in a cost-saving of US\$782 per baby (49).

The incorporation of oral dextrose gel into clinical practice has been evaluated in pre-and post-introduction observational studies in several parts of the world, with most reporting that oral dextrose was associated with a reduced NICU admission and increased breastfeeding (50-54). Its use is now recommended in several national guidelines $(15,22,24)$.

\section{Prophylaxis}

There is some evidence that even transient and undetected episodes of neonatal hypoglycemia may be associated with adverse sequelae. One study of 1,395 babies born in a center where glucose screening was universal showed that a single episode of transient neonatal hypoglycemia $[<35 \mathrm{mg} / \mathrm{dl}(1.9$ $\mathrm{mmol} / \mathrm{l})$ ] was associated with lower 4th-grade literacy and numeracy proficiency at 10 years of age (55). The Children With Hypoglycemia and Their Later Development (CHYLD) study demonstrated that clinically undetected low interstitial glucose concentrations were associated with an increased risk of executive dysfunction at 4.5 years of age (56). These findings suggest that even an effective treatment for neonatal hypoglycemia would not be sufficient to optimize outcomes for all babies, and prophylaxis needs to be considered.

The prophylactic measures currently recommended include early feeding, ensuring babies are warm and dry, and early skin-to-skin contact (57). These measures are thought to have a glucose sparing effect (58), but the evidence that they alter blood glucose concentrations or the incidence of hypoglycemia is limited (59-61).

Oral dextrose gel is being tested as an additional prophylactic measure to prevent hypoglycemia in at-risk babies. A dosefinding trial (Pre-hPOD) of 416 at-risk babies randomized to either placebo or dextrose gel at one of four different dosing schedules reported that a single dose of prophylactic oral $40 \%$ dextrose gel $(200 \mathrm{mg} / \mathrm{kg})$ in combination with breastfeeding was the most effective and practical dose (62), with a number needed to treat to prevent one case of hypoglycemia of 10. Further, the treatment was found to be acceptable, well tolerated, and had no adverse events (62). Follow-up at 2 years' corrected age showed no adverse effects, similar rates of neurosensory impairment between the groups, and a trend toward improved executive function scores in the dextrose gel group (63).

A quasi-experimental study of 236 at-risk babies reported that compared with feeding, prophylactic oral dextrose gel $200 \mathrm{mg} / \mathrm{kg}$ was not associated with a decreased incidence of hypoglycemia [ $<40 \mathrm{mg} / \mathrm{dl}(2.2 \mathrm{mmol} / \mathrm{l})]$ or admission to NICU (64). However, this study was not randomized, and the preparation used (Insta-Glucose gel) includes additional carbohydrates other than dextrose, which are likely to have competed with dextrose for membrane uptake and potentially reduced the effectiveness of this approach.

A multicenter randomized trial (hPOD) investigating whether prophylactic oral dextrose gel prevents neonatal hypoglycemia and hence reduces NICU admission has finished recruitment (ANZC Trials Registry - ACTRN12614001263684) (65). The results, and particularly the findings of the planned longterm follow-up, will provide valuable insight into whether prophylaxis with dextrose gel should be introduced into clinical practice.

\section{OUTCOMES OF NEONATAL HYPOGLYCEMIA}

Magnetic Resonance Imaging (MRI) studies have shown that neonatal hypoglycemia can cause brain injury $(66,67)$. The most widely reported pattern of acute brain injury is localized in the parietal and occipital regions (68), which are involved in visual processing. However, the evidence is inconsistent on whether neonatal hypoglycemia is associated with later visual problems (69). Injury may extend beyond these regions with reports of 
global or periventricular damage (67) as well as damage to the basal ganglia and thalamic regions $(67,70)$.

A systematic review and meta-analysis of six cohort studies with a sample size of 1,675 babies reported that neonatal hypoglycemia [definitions ranged from $<20-47 \mathrm{mg} / \mathrm{dl}$ (1.1$2.6 \mathrm{mmol} / \mathrm{l})$ ] was not associated with neurodevelopmental impairment, cognitive or motor deficits between 2 and 5 years of age (4). However, neonatal hypoglycemia was associated with a 3-fold increased risk of visual-motor impairment and executive dysfunction at 4 years of age. These risks were heightened for children who had experienced severe, recurrent or clinically undetected neonatal hypoglycemia (56). In older children, limited data (two studies, sample size of 54 babies) showed that neonatal hypoglycemia was associated with more than a 3-fold increased risk of neurodevelopmental impairment at 6-11 years of age, and a 2-fold increase in low numeracy and literacy (4). No studies reported on outcomes for adolescents.

Most of the evidence about long-term outcomes after neonatal hypoglycemia comes from retrospective observational studies, few of which have controlled for potential confounders or looked at outcomes beyond very early childhood. For example, infants of mothers with diabetes, who are at increased risk of neonatal hypoglycemia, have an increased risk of adverse outcomes (71, 72 ), but it is unclear how much of this risk is attributable to neonatal hypoglycemia. There is high heterogeneity between the studies which made comparing outcomes problematic, and there have been frequent calls for robust randomized trial evidence (3).

A randomized non-inferiority trial was the first to begin to address this major knowledge gap by comparing treatment at a threshold of $47 \mathrm{mg} / \mathrm{dl}(2.6 \mathrm{mmol} / \mathrm{l})$ against treatment at a lower threshold of $36 \mathrm{mg} / \mathrm{dl}(2.0 \mathrm{mmol} / \mathrm{l})$ among a sample of 689 otherwise healthy late preterm and term babies with mild-moderate hypoglycemia $[36 \mathrm{mg} / \mathrm{dl}(2.0 \mathrm{mmol} / \mathrm{l})-46$ $\mathrm{mg} / \mathrm{dl}(2.5 \mathrm{mmol} / \mathrm{l})]$ (73). Babies with early (birth to $2 \mathrm{~h}$ ) and severe $[\leq 35 \mathrm{mg} / \mathrm{dl}(1.9 \mathrm{mmol} / \mathrm{l})]$ hypoglycemia were excluded. In babies randomized to treatment at the lower threshold, fewer were monitored and treated, but there were more severe and recurrent hypoglycemic episodes ( $\geq 4$ episodes) compared with babies in the higher threshold group. Hospital costs

\section{REFERENCES}

1. Hay WW, Raju TNK, Higgins RD, Kalhan SC, Devaskar SU. Knowledge gaps and research needs for understanding and treating neonatal hypoglycemia: workshop report from Eunice Kennedy Shriver National Institute of Child Health and Human Development. J Pediatr. (2009) 155:6127. doi: $10.1016 /$ j.jpeds.2009.06.044

2. Harris D, Weston PJ, Harding JE. Incidence of neonatal hypoglycemia in babies identified as at risk. J Pediatr. (2012) 161:787-91. doi: 10.1016/j.jpeds.2012.05.022

3. Boluyt $\mathrm{N}$, van Kempen A, Offringa $M$. Neurodevelopment after neonatal hypoglycemia: a systematic review and design of an optimal future study. Pediatrics. (2006) 117:2231-43. doi: 10.1542/peds. 2005-1919

4. Shah R, Harding JE, Brown J, McKinlay C. Neonatal glycaemia and neurodevelopmental outcomes: a systematic review and meta-analysis. Neonatology. (2019) 115:116-26. doi: 10.1159/0004 92859 and duration of stay were similar between the groups, as were motor and cognitive functioning at 18 months on the Bayley Scales for Infant and Toddler Development. However, since previous studies have shown no relationship between neonatal hypoglycemia and motor or cognitive function at this age (4), this finding is not surprising, and the greater exposure to severe and recurrent hypoglycemia in the low threshold group is of concern. Much longer follow-up, at least to school age, will be essential to realize the true value of this important study.

\section{CONCLUSION}

Over the last few years, neonatal hypoglycemia has received much attention. However, what remains unclear is the extent to which transient asymptomatic neonatal hypoglycemia is associated with brain injury and neurodevelopmental impairment, and if so, at what glucose concentration maintained for how long. To address this, adequately powered randomized trials are needed of both prophylactic and treatment interventions at different glucose thresholds, with neurodevelopmental outcomes assessed at least to school age.

\section{AUTHOR CONTRIBUTIONS}

TE and $\mathrm{JH}$ contributed to the literature search and the design of the review. TE wrote the first draft of the manuscript and contributed to further revisions. $\mathrm{JH}$ contributed to the writing of the manuscript and supervised the study. Both authors contributed to the article and approved the submitted version.

\section{FUNDING}

This research was supported in part by grants from The Health Research Council of New Zealand (13/131, 15/216, 17/240) and the Eunice Kennedy Shriver National Institute of Child Health and Human Development (R01HD069622, 1RO1HD091075).

5. Schneider H, Reiber W, Sager R, Malek A. Asymmetrical transport of glucose across the in vitro perfused human placenta. Placenta. (2003) 24:2733. doi: 10.1053/plac.2002.0869

6. Holme AM, Roland MCP, Lorentzen B, Michelsen TM, Henriksen T. Placental glucose transfer: a human in vivo study. PLoS ONE. (2015) 10:e0117084. doi: 10.1371/journal.pone.0117084

7. Bozzetti P, Ferrari MM, Marconi AM, Ferrazzi E, Pardi G, Makowski EL, et al. The relationship of maternal and fetal glucose concentrations in the human from midgestation until term. Metabolism. (1988) 37:35863. doi: 10.1016/0026-0495(88)90136-9

8. Güemes M, Rahman SA, Hussain K. What is a normal blood glucose? Arch Dis Child. (2016) 101:569-74. doi: 10.1136/archdischild-2015-308336

9. Hillman NH, Kallapur SG, Jobe AH. Physiology of transition from intrauterine to extrauterine life. Clin Perinatol. (2012) 39:769-83. doi: 10.1016/j.clp.2012.09.009

10. Srinivasan G, Pildes RS, Cattamanchi G, Voora S, Lilien LD. Plasma glucose values in normal neonates: a new look. J Pediatr. (1986) 109:1147. doi: 10.1016/S0022-3476(86)80588-1 
11. Harris D, Weston PJ, Gamble GD, Harding JE. Glucose profiles in healthy term infants in the first 5 days: the Glucose in Well Babies (GLOW) Study. J Pediatr. (2020) 223:34-41e.4. doi: 10.1016/j.jpeds.2020.0 2.079

12. Sunehag AL, Haymond MW. Glucose extremes in newborn infants. Clin Perinatol. (2002) 29:245-60. doi: 10.1016/S0095-5108(02)00006-4

13. Harris D, Weston PJ, Harding JE. Lactate, rather than ketones, may provide alternative cerebral fuel in hypoglycaemic newborns. Arch Dis Child Fetal Neonatal Ed. (2015) 100:F161-4. doi: 10.1136/archdischild-2014-306435

14. Tin W. Defining neonatal hypoglycaemia: a continuing debate. Semin Fetal Neonatal Med. (2014) 19:27-32. doi: 10.1016/j.siny.2013.09.003

15. Clinical Practice Committee. Guidelines for the Management of Hypoglycaemia. (2018) Available online at: http://www.adhb.govt.nz/ newborn/Guidelines/Nutrition/HypoglycaemiaManagement.htm (accessed April 10, 2020).

16. Dixon KC, Ferris RL, Marikar D, Chong M, Mittal A, Manikam L, et al. Definition and monitoring of neonatal hypoglycaemia: a nationwide survey of NHS England Neonatal Units. Arch Dis Child Fetal Neonatal Ed. (2017) 102:F92-3. doi: 10.1136/archdischild-2016-311473

17. Harris D, Weston PJ, Battin MR, Harding JE. A survey of the management of neonatal hypoglycaemia within the Australian and New Zealand Neonatal Network. J Paediatr Child Health. (2014) 50:E55-62. doi: 10.1111/j.1440-1754.2009.01599.x

18. Lucas A, Morley R, Cole TJ. Adverse neurodevelopmental outcome of moderate neonatal hypoglycaemia. BMJ. (1988) 297:1304-8. doi: 10.1136/bmj.297.6659.1304

19. Lucas A, Morley R. Authors' reply to cornblath - outcome of neonatal hypoglycaemia. Complete data are needed. Br Med J. (1999) 318:195. doi: 10.1136/bmj.318.7177.194a

20. Koh TH, Aynsley-Green A, Tarbit M, Eyre JA. Neural dysfunction during hypoglycaemia. Arch Dis Child. (1988) 63:1353-8. doi: 10.1136/adc.63.11.1353

21. Adamkin DH, Committee on Fetus and Newborn. Postnatal glucose homeostasis in late-preterm and term infants. Pediatrics. (2011) 127:5759. doi: 10.1542/peds.2010-3851

22. Canadian Paediatric Society. Screening guidelines for newborns at risk for low blood glucose. Paediatr Child Health. (2004) 9:723-9. doi: 10.1093/pch/9.10.723

23. Cornblath M, Hawdon JM, Williams AF, Aynsley-Green A, Ward-Platt MP, Schwartz R, et al. Controversies regarding definition of neonatal hypoglycemia: suggested operational thresholds. Pediatrics. (2000) 105:11415. doi: 10.1542/peds.105.5.1141

24. British Association of Perinatal Medicine. Identification and Management of Neonatal Hypoglycaemia in the Full Term Infant - A Framework for Practice. (2017). p. 1-31. Available online at: https://www.bapm.org/resources/40identification-and-management- of-neonatal-hypoglycaemia-in-the-fullterm-infant-2017 (accessed April 23, 2020).

25. Harding JE, Harris D, Hegarty JE, Alsweiler JM, McKinlay CJD. An emerging evidence base for the management of neonatal hypoglycaemia. Early Hum Dev. (2017) 104:51-6. doi: 10.1016/j.earlhumdev.2016.12.009

26. Bromiker R, Perry A, Kasirer Y, Einav S, Klinger G, Levy-Khademi F. Early neonatal hypoglycemia: incidence of and risk factors. A cohort study using universal point of care screening. J Matern Neonatal Med. (2019) 32:78692. doi: 10.1080/14767058.2017.1391781

27. Thornton PS, Stanley CA, De Leon DD, Harris D, Haymond MW, Hussain K, et al. Recommendations from the Pediatric Endocrine Society for evaluation and management of persistent hypoglycemia in neonates, infants, and children. J Pediatr. (2015) 167:238-45. doi: 10.1016/j.jpeds.2015.0 3.057

28. Adamkin DH. Neonatal hypoglycemia. In: Martin G, Rosenfield W, editors. Common Problems in the Newborn Nursery. An Evidence Case-Based Guide. Basel: Springer (2019). p. 99-108.

29. Beardsall K. Measurement of glucose levels in the newborn. Early Hum Dev. (2010) 86:263-7. doi: 10.1016/j.earlhumdev.2010.05.005

30. Williams AF. Hypoglycaemia of the newborn: a review. Bull World Health Organ. (1997) 75:261-90.

31. Sreenivasa B, Kumar G V, Sreenivasa B. Comparative study of blood glucose levels in neonates using glucometer and laboratory glucose oxidase method. Curr Pediatr Res. (2015) 19:29-32.
32. Khan AI, Vasquez Y, Gray J, Wians Jr FH, Kroll MH. The variability of results between point-of-care testing glucose meters and the central laboratory analyzer. Arch Pathol Lab Med. (2006) 130:1527-32. doi: 10.1043/1543-2165(2006)1301527:TVORBP2.0.CO;2

33. Glasgow MJ, Harding JE, Edlin R. Cost analysis of cotside screening methods for neonatal hypoglycaemia. Neonatology. (2018) 114:155-62. doi: 10.1159/00048 9080

34. McKinlay CJD, Chase JG, Dickson J, Harris D, Alsweiler JM, Harding JE. Continuous glucose monitoring in neonates: a review. Matern Heal Neonatol Perinatol. (2017) 3:1-9. doi: 10.1186/s40748-017-0055-z

35. Signal M, Le Compte A, Harris D, Weston PJ, Harding JE. Impact of retrospective calibration algorithms on hypoglycemia detection in newborn infants using continuous glucose monitoring. Diabetes Technol Ther. (2012) 14:883-90. doi: 10.1089/dia.2012.0111

36. Shah R, McKinlay CJD, Harding JE. Neonatal hypoglycemia: continuous glucose monitoring. Curr Opin Pediatr. (2018) 30:204-8. doi: 10.1097/MOP.0000000000000592

37. Harris D, Battin MR, Williams CE, Weston PJ, Harding JE. Cot-side electro-encephalography and interstitial glucose monitoring during insulininduced hypoglycaemia in newborn lambs. Neonatology. (2009) 95:2718. doi: $10.1159 / 000166847$

38. Beardsall K, Ogilvy-Stuart AL, Ahluwalia J, Thompson M, Dunger DB. The continuous glucose monitoring sensor in neonatal intensive care. Arch Dis Child Fetal Neonatal Ed. (2005) 90:F307-10. doi: 10.1136/adc.2004. 051979

39. Harris D, Battin MR, Weston PJ, Harding JE. Continuous glucose monitoring in newborn babies at risk of hypoglycemia. J Pediatr. (2010) 157:198202. doi: 10.1016/j.jpeds.2010.02.003

40. Uettwiller F, Chemin A, Bonnemaison E, Favrais G, Saliba E, Labarthe F. Real-time continuous glucose monitoring reduces the duration of hypoglycemia episodes: a randomized trial in very low birth weight neonates. PLoS ONE. (2015) 10:e0116255. doi: 10.1371/journal.pone. 0116255

41. Galderisi A, Facchinetti A, Steil GM, Ortiz-Rubio P, Cavallin F, Tamborlane WV, et al. Continuous glucose monitoring in very preterm infants: a randomized controlled trial. Pediatrics. (2017) 140:1-10. doi: 10.1542/peds.2017-1162

42. Hoe FM, Thornton PS, Wanner LA, Steinkrauss L, Simmons RA, Stanley CA. Clinical features and insulin regulation in infants with a syndrome of prolonged neonatal hyperinsulinism. J Pediatr. (2006) 148:20712. doi: 10.1016/j.jpeds.2005.10.002

43. Miralles RE, Lodha A, Perlman M, Moore AM. Experience with intravenous glucagon infusions as a treatment for resistant neonatal hypoglycemia. Arch Pediatr Adolesc Med. (2002) 156:999-1004. doi: 10.1001/archpedi.156.10.999

44. Sweet CB, Grayson S, Polak M. Management strategies for neonatal hypoglycemia. J Pediatr Pharmacol Ther. (2013) 18:199-208. doi: 10.5863/1551-6776-18.3.199

45. Rozance PJ, Wolfsdorf JI. Hypoglycemia in the newborn. Pediatr Clin North Am. (2019) 66:333-42. doi: 10.1016/j.pcl.2018.12.004

46. Weston PJ, Harris D, Battin M, Brown J, Hegarty JE, Harding JE. Oral dextrose gel for the treatment of hypoglycaemia in newborn infants. Cochrane Database Syst Rev. (2016) CD011027. doi: 10.1002/14651858.CD011027.pub2

47. Harris D, Weston PJ, Signal M, Chase JG, Harding JE. Dextrose gel for neonatal hypoglycaemia (the Sugar Babies Study): a randomised, double-blind, placebo-controlled trial. Lancet. (2013) 382:2077-83. doi: 10.1016/S0140-6736(13)61645-1

48. Harris D, Alsweiler JM, Ansell JM, Gamble GD, Thompson B, Wouldes $\mathrm{TA}$, et al. Outcome at 2 years after dextrose gel treatment for neonatal hypoglycaemia: follow-up of a randomized trial. J Pediatr. (2016) 170:549. doi: 10.1016/j.jpeds.2015.10.066

49. Glasgow MJ, Harding JE, Edlin R, Alsweiler JM, Chase JG, Harris D, et al. Cost analysis of treating neonatal hypoglycemia with dextrose gel. J Pediatr. (2018) 198:151-5.e1. doi: 10.1016/j.jpeds.2018.02.036

50. Gregory K, Turner D, Benjamin CN, Monthe-Dreze C, Johnson L, Hurwitz $S$, et al. Incorporating dextrose gel and feeding in the treatment of neonatal hypoglycaemia. Arch Dis Child Fetal Neonatal Ed. (2020) 105:459. doi: 10.1136/archdischild-2018-316430 
51. Rawat M, Chandrasekharan P, Turkovich S, Barclay N, Perry K, Schroeder $\mathrm{E}$, et al. Oral dextrose gel reduces the need for intravenous dextrose therapy in neonatal hypoglycemia. Biomed Hub. (2016) 1:1-9. doi: 10.1159/0004 48511

52. Bennett C, Fagan E, Chaharbakhshi E, Zamfirova I, Flicker J. Implementing a protocol using glucose gel to treat neonatal hypoglycemia. Nurs Womens Health. (2016) 20:64-74. doi: 10.1016/j.nwh.2015. 11.001

53. Makker K, Alissa R, Dudek C, Travers L, Smotherman C, Hudak ML. Glucose gel in infants at risk for transitional neonatal hypoglycemia. Am J Perinatol. (2018) 35:1050-6. doi: 10.1055/s-0038-1639338

54. Stewart CE, Sage ELM, Reynolds P. Supporting 'baby friendly': a quality improvement initiative for the management of transitional neonatal hypoglycaemia. Arch Dis Child Fetal Neonatal Ed. (2016) 101:F3447. doi: 10.1136/archdischild-2015-308950

55. Kaiser JR, Bai S, Gibson N, Holland G, Lin TM, Swearingen CJ, et al. Association between transient newborn hypoglycemia and fourth-grade achievement test proficiency: a population-based study. JAMA Pediatr. (2015) 169:913-21. doi: 10.1001/jamapediatrics.2015.1631

56. McKinlay CJD, Alsweiler JM, Anstice NS, Burakevych N, Chakraborty A, Chase JG, et al. Association of neonatal glycemia with neurodevelopmental outcomes at 4.5 years. JAMA Pediatr. (2017) 171:972-83. doi: 10.1001/jamapediatrics.2017.1579

57. UNICEF. Guidance on the Development of Policies and Guidelines for the Prevention and Management of Hypoglycaemia of the Newborn. (2013). Available online at: https://www.unicef.org.uk/babyfriendly/wpcontent/uploads/sites/2/2010/10/hypo_policy.pdf (accessed April 27, 2020).

58. Moore ER, Bergman N, Anderson GC, Medley N. Early skin-to-skin contact for mothers and their healthy newborn infants. Cochrane Database Syst Rev. (2016). doi: 10.1002/14651858.CD003519.pub4

59. Sweet DG, Hadden D, Halliday HL. The effect of early feeding on the neonatal blood glucose level at 1-hour of age. Early Hum Dev. (1999) 55:636. doi: 10.1016/S0378-3782(99)00004-3

60. Chiruvolu A, Miklis KK, Stanzo KC, Petrey B, Groves CG, McCord $\mathrm{K}$, et al. Effects of skin-to-skin care on late preterm and term infants at-risk for neonatal hypoglycemia. Pediatr Qual Saf. (2017) 2:1-7. doi: 10.1097/pq9.0000000000000030

61. Chertok IR, Raz I, Shoham I, Haddad H, Wiznitzer A. Effects of early breastfeeding on neonatal glucose levels of term infants born to women with gestational diabetes. J Hum Nutr Diet. (2009) 22:1669. doi: 10.1111/j.1365-277X.2008.00921.x

62. Hegarty JE, Harding JE, Gamble GD, Crowther CA, Edlin R, Alsweiler JM. Prophylactic oral dextrose gel for newborn babies at risk of neonatal hypoglycaemia: a randomised controlled dose-finding trial (the PrehPOD Study). PLoS Med. (2016) 13:e1002155. doi: 10.1371/journal.pmed. 1002155

63. Griffith R, Hegarty JE, Alsweiler JM, Gamble GD, May R, McKinlay CJD, et al. Two-year outcomes after dextrose gel prophylaxis for neonatal hypoglycaemia. Arch Dis Childh Fetal Neonatal Ed. (2020). doi: 10.1136/archdischild-2020-320305. [Epub ahead of print].
64. Coors SM, Cousin JJ, Hagan JL, Kaiser JR. Prophylactic dextrose gel does not prevent neonatal hypoglycemia: a quasi-experimental pilot study. J Pediatr. (2018) 198:156-61. doi: 10.1016/j.jpeds.2018.02.025

65. Harding JE, Hegarty JE, Crowther CA, Edlin R, Gamble G, Alsweiler JM. Randomised trial of neonatal hypoglycaemia prevention with oral dextrose gel (hPOD): study protocol. BMC Pediatr. (2015) 15:1-5. doi: 10.1186/s12887-015-0440-6

66. Filan PM, Inder TE, Cameron FJ, Kean MJ, Hunt RW. Neonatal hypoglycemia and occipital cerebral injury. J Pediatr. (2006) 148:5525. doi: 10.1016/j.jpeds.2005.11.015

67. Burns CM, Rutherford MA, Boardman JP, Cowan FM. Patterns of cerebral injury and neurodevelopmental outcomes after symptomatic neonatal hypoglycemia. Pediatrics. (2008) 122:65-74. doi: 10.1542/peds.2007-2822

68. Alkalay AL, Flores-Sarnat L, Sarnat HB, Moser FG, Simmons CF. Brain imaging findings in neonatal hypoglycemia: case report and review of 23 cases. Clin Pediatr. (2005) 44:783-90. doi: 10.1177/000992280504400906

69. Paudel N, Chakraborty A, Anstice N, Jacobs RJ, Hegarty JjE, Harding JE, et al. Neonatal hypoglycaemia and visual development: a review. Neonatology. (2017) 112:47-52. doi: 10.1159/000456705

70. Gataullina S, Lonlay PDE, Dellatolas G, Valayannapoulos V, Napuri S, Damaj L, et al. Topography of brain damage in metabolic hypoglycaemia is determined by age at which hypoglycaemia occurred. Dev Med Child Neurol. (2013) 55:162-6. doi: 10.1111/dmcn.12045

71. Lin C-H, Lin W-D, Chou IC, Lee I-C, Hong S-Y. Infants of mothers with diabetes and subsequent attention deficit hyperactivity disorder: a retrospective cohort study. Front Pediatr. (2019) 7:1-7. doi: 10.3389/fped.2019.00452

72. Pacaud D, Dewey D. Neurocognitive outcome of children exposed to severe hypoglycemia in utero. Diabetes Manag. (2011) 1:129-40. doi: 10.2217/dmt.10.10

73. van Kempen AA, Eskes PF, Nuytemans DHGM, van der Lee JH, Dijksman LM, van Veenendaal NR, et al. Lower versus traditional treatment threshold for neonatal hypoglycemia. N Engl J Med. (2020) 382:53444. doi: 10.1056/NEJMoa1905593

Disclaimer: The content is solely the responsibility of the authors and does not necessarily represent the official views of the Eunice Kennedy Shriver National Institute of Child Health and Human Development or the National Institutes of Health.

Conflict of Interest: The authors declare that the research was conducted in the absence of any commercial or financial relationships that could be construed as a potential conflict of interest.

Copyright (c) 2021 Edwards and Harding. This is an open-access article distributed under the terms of the Creative Commons Attribution License (CC BY). The use, distribution or reproduction in other forums is permitted, provided the original author(s) and the copyright owner(s) are credited and that the original publication in this journal is cited, in accordance with accepted academic practice. No use, distribution or reproduction is permitted which does not comply with these terms. 